\title{
Discrepancy of Breast and Axillary Pathologic Complete Response and Outcomes in Different Subtypes of Node-positive Breast Cancer after Neoadjuvant Chemotherapy
}

\author{
Shin-Cheh Chen ${ }^{1 * 凶}$, Chi-Chang Yu ${ }^{1 *}$, Hsien-Kun Chang ${ }^{2}$, Yung-Chang Lin², Yung-Feng Lo' ${ }^{1}$, Shih-Che \\ Shen ${ }^{1}$, Wen-Lin Kuo ${ }^{1}$, Hsiu-Pei Tsai ${ }^{1}$, Hsu-Huan Chou ${ }^{1}$, Chia-Hui Chu' ${ }^{1}$, Wen-Chi Shen ${ }^{2}$, Ren-Chin Wu ${ }^{3}$, \\ Shir-Hwa Ueng 3 , Yi-Ting Huang ${ }^{4}$ \\ 1. Department of General Surgery, Chang Gung Memorial Hospital at Linkou, Chang Gung University College of Medicine, Taoyuan, Taiwan \\ 2. Department of Hematology-Oncology, Chang Gung Memorial Hospital at Linkou, Chang Gung University College of Medicine, Taoyuan, Taiwan \\ 3. Department of Pathology, Chang Gung Memorial Hospital at Linkou, Chang Gung University College of Medicine, Taoyuan, Taiwan \\ 4. Department of Radiation Oncology, Chang Gung Memorial Hospital at Linkou, Chang Gung University Medical College, Taoyuan, Taiwan \\ *Shin-Cheh Chen and Chi-Chang Yu contribute equally to this work
}

$\triangle$ Corresponding author: Shin-Cheh Chen, MD, Department of General Surgery, Chang Gung Memorial Hospital at Linkou, Chang Gung University College of Medicine, Taoyuan, Taiwan, No.5, Fuxing St., Guishan Dist., Taoyuan City 333, Taiwan (R.O.C.). Telephone: +886-3-3281200 Ext. 3234; Fax: +886-3-3285818

(C) The author(s). This is an open access article distributed under the terms of the Creative Commons Attribution License (https://creativecommons.org/licenses/by/4.0/). See http://ivyspring.com/terms for full terms and conditions.

Received: 2021.05.17; Accepted: 2021.06.24; Published: 2021.07.06

\begin{abstract}
Few studies have analyzed the discrepancy between breast pathologic complete response (B-pCR) and axillary node $P C R(N-p C R)$ rates and their impact on survival outcomes in different intrinsic subtypes of early breast cancer after neoadjuvant chemotherapy (NAC). We retrospectively reviewed B-pCR, N-pCR, and total (breast and axillary node) pCR (T-pCR) after NAC to assess the discrepancy and outcomes between 2005 and 2017. A total of 968 patients diagnosed with cT1-4c, N1-2, and M0 breast cancer were enrolled in the study. The median age was 49 years and the median follow-up time was 45 months. Of these patients, 213 achieved T-pCR, 31 achieved B-pCR with axillary node pathologic non-complete response ( $N$-non $P C R$ ), 245 achieved $N$-pCR with breast pathologic non-complete response (B-non $P C R$ ), and 479 achieved total (breast and axillary node) pathologic non-complete response (T-non $p C R$ ) after NAC. The highest $B-p C R$ and N-pCR rates were found in the hormone receptor-negative, human epidermal growth factor receptor 2-positive $\mathrm{HR}(-) \mathrm{HER} 2(+)$ subtype, while the lowest B-pCR rate was found in the HR(+)HER2(-) subtype. The $N-p C R$ rate was correlated to the $B-p C R$ rate $(P<0.001)$, but was higher than the $B-p C R$ rate in all subtypes. The 5 -year overall survival $(O S)$ rates for patients with T-pCR, B-pCR, and N-pCR were $91.2 \%, 91.7 \%$, and $91.9 \%$, respectively. For non-pCR, non-pCR, and non-pCR, the 5 -year OS rates were $73.6 \%, 78.9 \%$, and $74.7 \%$, respectively $(\mathrm{P}<0.0001)$. B-non $P C R$ patients had a lower risk of recurrence than $\mathrm{T}$-non $\mathrm{PCR}$ or $\mathrm{N}$-non-pCR patients, although there were no differences in OS among them. In conclusion, the $N$-pCR rate was higher than the $B-p C R$ rate after NAC in all intrinsic subtypes, and N-non PCR or T-non $p C R$ patients had the worst outcomes.
\end{abstract}

Key words: breast cancer, neoadjuvant chemotherapy, pathologic complete response, intrinsic subtype

\section{Introduction}

Neoadjuvant chemotherapy (NAC) is a treatment strategy for increasing the rate of breast-conserving surgery in operable breast cancer and increasing operability in inoperable breast cancer, especially in triple-negative and human epidermal growth factor receptor 2 (HER2) overexpression subtypes. Pathologic complete response (pCR) rates vary among different subtypes but are considered the 
most important surrogate markers of disease-free survival (DFS) and overall survival (OS). However, some contradictory meta-analyses have been reported.[1-7] High pCRs of the breast tumor itself (ypT0/is, B-pCR) and axillary lymph nodes (ypN0, $\mathrm{N}$-pCR) have been proposed to be linked to the omission of primary surgery after minimally invasive biopsy [8] and de-escalation axillary dissection by sentinel node biopsy.[9]

The discrepancy between B-pCR and N-pCR rates in clinically node-positive $(\mathrm{cN}+)$ disease has rarely been reported, except for tumor heterogeneity as the underlying biological event to explain the difference in primary disease and metastatic nodes.[10] N-pCR rates were reported to be higher than $\mathrm{B}-\mathrm{pCR}$ rates in $\mathrm{cN}+$ patients, although inconsistent results of $\mathrm{N}-\mathrm{pCR}$ rates have been reported in various intrinsic subtypes of $\mathrm{cN}+$ disease.[7, 11, 12] There are many predictive models to identify N-pCR for omission of axillary surgery, although this is not practical.[13-15]

Moreover, only a few studies have discussed the impact of B-pCR or N-pCR separately on survival benefit after NAC $[7,8,13,15]$ and there are only a limited number of trials that have defined the impact of non-pCR either in the breast or axillary node on survival.[16] Nevertheless, residual nodal disease is considered a more important prognostic factor of survival than residual breast burden. [11]

In this study, we analyzed the differences between total (breast and axillary node) pCR (T-pCR), $\mathrm{B}-\mathrm{pCR}$, and $\mathrm{N}$-pCR rates in $\mathrm{cN}+$ breast cancer after NAC in various intrinsic subtypes. Further, exploring the role of B-pCR and/or N-pCR contributes to the survival benefit and impact of non-pCR either in the breast or axilla nodes on outcomes in different intrinsic subtypes.

\section{Material and methods}

We conducted a retrospective review of all breast cancer patients treated with NAC between January 2005 and December 2017 at our institution. The inclusion criteria consisted of women aged 20 years or older who (1) had histologically proven clinical stage T1 through T4c, N1 through N2, M0 primary invasive breast cancer according to the eight edition of the American Joint Commission on Cancer TNM staging system, (2) had axillary nodal disease confirmed by fine-needle aspiration or core-needle biopsy, and (3) had underwent curative surgery after NAC. The exclusion criteria consisted of patients who (1) had synchronous bilateral breast cancer, (2) had clinically node-negative (cN0) status, (3) had inflammatory breast cancer, and (4) had distant metastases. In our institution, axillary nodal status was routinely determined before NAC administration using axillary ultrasound. If axillary ultrasound showed no suspicious lymph nodes, the patient was defined as cN0. The patients were defined as $\mathrm{cN}+$, while suspicious lymph nodes were confirmed with additional fine-needle aspiration cytology or core-needle biopsy. cN1 was defined as metastasis to movable ipsilateral level I and/or level II axillary nodes, and cN2 was defined as metastasis to fixed or matted ipsilateral level I and/or level II axillary lymph nodes. The administered NAC regimens included the following: four cycles of TE (docetaxel, epirubicin); or four cycles of fluorouracil, epirubicin, and cyclophosphamide followed by four cycles of docetaxel. In the case of HER2(+) breast cancer, trastuzumab was recommended as targeted therapy in addition to chemotherapy and continued for 1 year. No HER2-targeted therapy was advised from 2005-2010.

HER2 status was evaluated by immunohistochemistry (IHC). An IHC score of 0 or $1+$ was considered negative. In the case of a $2+$ IHC score, fluorescence in situ hybridization (FISH) was mandatory in addition to IHC. An IHC score of 3 $(>10 \%$ of cells with strong intensity circumferential membrane staining) or FISH positivity was defined as positive. Hormonal receptor (HR) positivity was defined as an IHC score of estrogen receptor or progesterone receptor $>1 \%$.

The cohort of patients was categorized into four intrinsic subtypes based on combinations of HR and HER2 status: HR(+)HER2(-), HR(+)HER2(+), HR(-)HER2(+), and HR(-)HER2(-). pCR was defined as the absence of residual invasive carcinoma in the surgical specimen. Response to NAC was categorized as B-pCR (ypT0/is) as no invasive carcinoma in the breast after NAC, N-pCR (ypN0) as no invasive carcinoma in the axillary node after NAC, T-pCR (ypT0/is ypN0) as no invasive carcinoma in both the breast and axillary nodes after NAC, B-non pCR as invasive carcinoma in the breast after NAC, N-non pCR as invasive carcinoma in the axillary node after NAC, and T-non pCR as invasive carcinoma in both the breast and axillary nodes. This study was approved by the Institutional Review Board of Chang Gung Memorial Hospital (IRB/CGMH) in Taiwan (IRB No. 201601335B0).

Chi-square tests and analysis of variance were used to assess the differences in categorical and continuous variables, proportions, and median values with interquartile ranges (IQRs). Six post-hoc tests with Bonferroni correction with $\mathrm{a}=0.05$ were simultaneously performed, and the correction was $0.05 / 6=0.00833$. A logistic regression model was used to estimate the associations between subtypes and 
post-NAC responses after adjusting for clinicopathologic characteristics. DFS was defined as the time from diagnosis to the date of breast cancer recurrence, the date of death from any cause, or the date of the last follow-up. OS was defined as the time from diagnosis to the date of death or last follow-up. Kaplan-Meier (KM) curves were used to visualize unadjusted OS for the entire cohort, and receptor subtype, with log-rank $\mathrm{P}<0.05$, was defined as significant. Cox proportional hazards modeling was used to estimate the association between subtypes and post-NAC response. The hazard ratios and 95\% confidence intervals (95\% CIs) with two-tailed $\mathrm{P}<0.05$ were considered significant. All analyses were conducted using SPSS version 20.0 (Statistical Package for the Social Sciences Version 20.0 IBM corp. Armonk, NY).

\section{Results}

A total of 968 patients were included in this analysis. Clinical features are summarized in Table 1. The median age at diagnosis was 49 (IQR: 14.5) years, and the average tumor size was 4.2 (IQR: 2.4$) \mathrm{cm}$. The median follow-up time was 45 (range, 5.1-163.2) months. There were $543(56.1 \%)$ patients categorized as having $\mathrm{cN} 1$ disease, and the remaining 425 (43.9\%) had cN2 disease. The distribution of intrinsic subtypes according to HR and HER2 status was as follows: 382 (39.5\%) patients were HR(+)HER2(-), $222(22.9 \%)$ were HR(+)HER2(+), $216(22.3 \%)$ were HR(-)HER2(+), and 148 (15.3\%) were HR(-)HER2(-). Anthracycline and taxanes were included in the treatment regimens for all subtypes, but using different schedules. Cisplatin combined with taxanes was used in 93 (63\%) HR(-)HER2(-) patients. Trastuzumab alone was administered to $242(55.3 \%)$ HER2(+) patients and dual blockade with trastuzumab plus pertuzumab was administered to $66(15.1 \%)$ HER2(+) patients.

There were $213(22.0 \%)$ patients who achieved T-pCR, 31 (3.2\%) patients achieved B-pCR with N-non pCR, $245(25.3 \%)$ patients achieved N-pCR with B-non-pCR, and 479 (49.5\%) patients achieved T-non pCR. In this cohort, we found a significantly higher N-pCR rate (458 patients, $47.3 \%)$ than B-pCR (244 patients, $25.2 \%$ ). The $\mathrm{pCR}$ and non-pCR rates according to subtype are shown in Table 2 . The lowest B-pCR rate (29 patients, $7.6 \%$ ) was found in the $\mathrm{HR}(+)$ HER2(-) subtype, while the highest B-pCR rate (95 patients, $44.0 \%)$ in HR(-)HER2(+) and highest N-pCR (133 patients, $61.6 \%$ ) were found in the HR(-)HER2(+) subtype. The N-pCR rate was highly correlated with B-pCR $(P<0.0001)$, and even higher rates (the differences: $22.5 \%-24.8 \%)$ were found in all molecular subtypes except HR(-)HER2(+) (difference: 17.6\%). In B-non-pCR patients, the N-pCR rates were $25.2 \%$,
$43.0 \%, 39.7 \%$, and $43.4 \%$ for HR(+)HER2(-), HR(+)HER2(+), HR(-)HER2(+), and HR(-)HER2(-) subtypes, respectively. In contrast, in N-non-pCR patients, the B-pCR rates were $10.3 \%, 14.1 \%, 10.5 \%$, and $16.3 \%$ in $\operatorname{HR}(+) \operatorname{HER} 2(-), \operatorname{HR}(+) \operatorname{HER}(+)$, HR(-)HER(+), and HR(-)HER2(-) subtypes, respectively. A relatively higher N-pCR rate in B-pCR patients in comparison to B-non $\mathrm{pCR}$ was found either in $\mathrm{cN} 1$ or $\mathrm{cN} 2$ diseases. Very high N-pCR rates (range, $86.1 \%-92.6 \%$ ) were found in B-pCR patients with cN1 disease in all subtypes (Supplementary Table 1A, 1B).

Table 1. Patients' clinicopathological and treatment characteristics

\begin{tabular}{ll}
\hline Clinical features & No. of cases (\%) \\
\hline No. of patients & 968 \\
Age (years), median (IQR) & $49(14.5)$ \\
Tumor size (cm), median (IQR) & $4.2(2.4)$ \\
Clinical T stage & \\
T1 & $39(4.0)$ \\
T2 & $519(53.7)$ \\
T3 & $192(19.8)$ \\
T4 & $218(22.5)$ \\
Clinical nodal status & $543(56.1)$ \\
N1 & $425(43.9)$ \\
N2 & \\
Histology & $952(98.4)$ \\
Invasive ductal carcinoma & $2(0.2)$ \\
Mucinous carcinoma & $10(1.0)$ \\
Invasive lobular carcinoma & $4(0.1)$ \\
Invasive micropapillary carcinoma & \\
Histologic grade & $69(7.1)$ \\
1 & $371(38.3)$ \\
2 & $440(45.5)$ \\
3 & $88(9.1)$ \\
Unknown & \\
ER status & $383(39.6)$ \\
Negative & $585(60.4)$ \\
Positive & \\
PR status & $484(50.0)$ \\
Negative & $484(50.0)$ \\
Positive & \\
HER2 status & $530(54.8)$ \\
Negative & $438(45.2)$ \\
Positive & \\
Subtype & \\
HR(-) HER2(+) & $216(22.3)$ \\
HR(+) HER2(-) & $382(39.5)$ \\
HR(+) HER2(+) & $222(22.9)$ \\
HR(-) HER2(-) & $148(15.3)$ \\
Operation type & \\
Mastectomy & $594(61.4)$ \\
Breast conserving surgery & $374(38.6)$ \\
Neoadjuvant regimens & \\
Antracycline + taxane & $471(48.7)$ \\
Antracycline + taxane + cisplatin & $189(19.5)$ \\
Antracycline + taxane + herceptin & $242(25.0)$ \\
Antracycline + taxane + herceptin + perjeta & $66(6.8)$ \\
Adjuvant hormone therapy & \\
No & \\
Yes & \\
Adjuvant radiotherapy & \\
No & \\
Yes & \\
\hline Abbreviations: IQR: interquartile range; ER: estrogen receptor; PR: progesterone \\
receptor; HER2: human epidermal growth factor receptor 2. \\
\end{tabular}




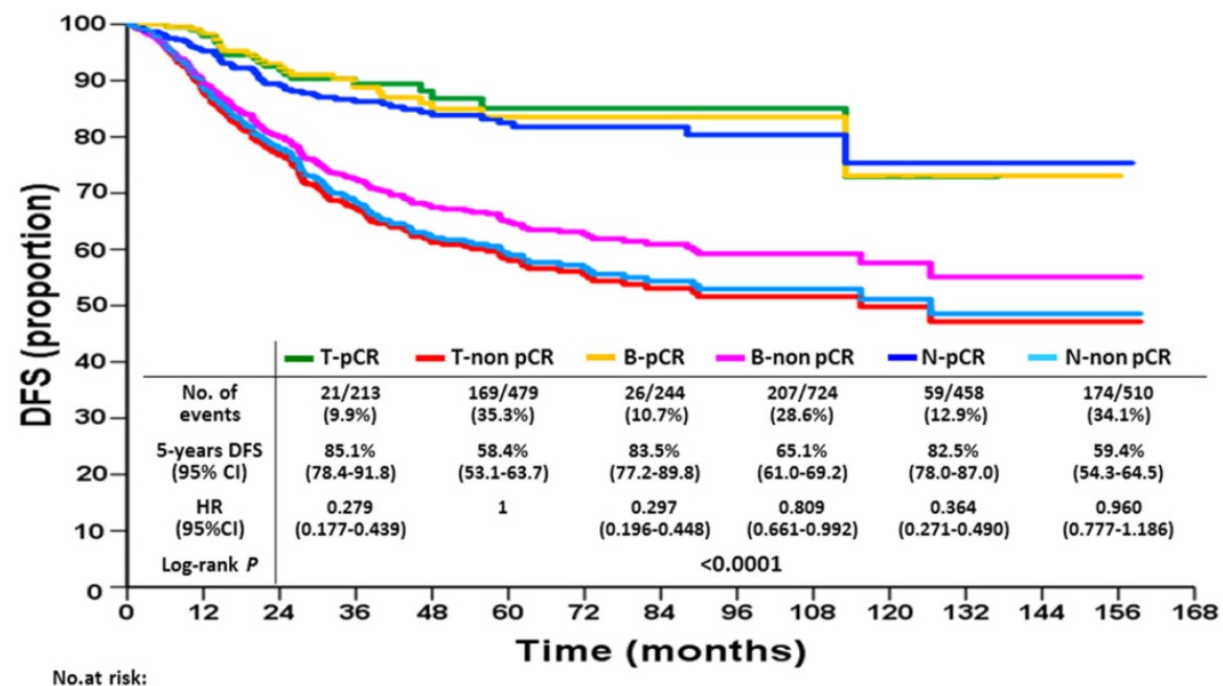

$\begin{array}{lcccccccccccccc}\text { - T-pCR } & 181 & 129 & 96 & 66 & 45 & 33 & 27 & 17 & 11 & 6 & 1 & 0 & 0 & 0 \\ \text { - T-nonpCR } & 369 & 279 & 217 & 172 & 137 & 97 & 76 & 67 & 38 & 22 & 16 & 9 & 2 & 0 \\ \text { - B-pCR } & 209 & 150 & 112 & 80 & 56 & 41 & 32 & 19 & 12 & 7 & 2 & 1 & 0 & 0 \\ \text { - B-nonpCR } & 557 & 424 & 334 & 263 & 209 & 154 & 116 & 94 & 53 & 28 & 19 & 10 & 3 & 0 \\ \text { - N-pCR } & 369 & 274 & 213 & 157 & 117 & 90 & 67 & 44 & 26 & 12 & 4 & 1 & 1 & 0 \\ \text { - N-nonpCR } & 397 & 300 & 233 & 186 & 148 & 105 & 81 & 69 & 39 & 23 & 17 & 10 & 3 & 0\end{array}$

Figure 1. Disease-free survival according to breast or axillary node response status after neoadjuvant chemotherapy

Table 2. $p C R$ and non-pCR rates of breast or axillary node after neoadjuvant chemotherapy $(\mathrm{cNl}, 2)$

\begin{tabular}{lllll}
\hline Subtypes & & $\begin{array}{l}\text { B-pCR, }(\mathrm{n}=244) \\
\text { no. }(\%)\end{array}$ & $\begin{array}{l}\text { B-non pCR, }(\mathrm{n}=724) \\
\text { no. }(\%)\end{array}$ & $p$ \\
\hline $\begin{array}{l}\text { HR(-) HER2(+) } \\
(\mathrm{n}=216)\end{array}$ & N-pCR & $85(89.5)$ & $48(39.7)$ & $<0.001$ \\
HR(+) HER2(-) & N-non pCR & $10(10.5)$ & $73(60.3)$ & $<0.001$ \\
$(\mathrm{n}=382)$ & N-non pCR & $26(89.7)$ & $89(25.2)$ & $<0.001$ \\
HR(+) HER2(+) & N-pCR & $61(85.9)$ & $264(74.8)$ & \\
$(\mathrm{n}=222)$ & N-non pCR & $10(14.1)$ & $65(43.0)$ & $<0.001$ \\
HR(-) HER2(-) & N-pCR & $41(83.7)$ & $43(57.0)$ & \\
$(\mathrm{n}=148)$ & N-non pCR & $8(16.3)$ & $56(56.6)$ & \\
\hline
\end{tabular}

Abbreviations: pCR: pathological complete response; B-pCR: breast pathologic complete response; B-non pCR: breast pathologic non-complete response; HR hormone receptor; HER2: human epidermal growth factor receptor $2 ; \mathrm{N}-\mathrm{pCR}$ : axillary node pathologic complete response; N-non pCR: axillary node pathologic non-complete response.

KM DFS and OS curves according to different response statuses are shown in Figures 1 and 2. The 5 -year DFS rates for patients with T-pCR, B-pCR, and N-pCR were 85.1\% (95\% CI, 78.4\%-91.8\%), 83.5\% (95\% CI, $77.2 \%-89.8 \%$ ), and $82.5 \%$ (95\% CI, $78.0 \%-87.0 \%)$, respectively. For T-non pCR, B-non pCR, and N-non pCR, it was 58.4\% (95\% CI, 53.1\%-63.7\%), 65.1\% (95\% CI, $61.0 \%-69.2 \%$ ), and $59.4 \%$ (95\% CI, 54.3\%-64.5\%), respectively $(\mathrm{P}<0.0001)$. The 5-year OS rates for patients with T-pCR, B-pCR, and N-pCR were 91.2\% (95\% CI, 85.5\%-96.9\%), 91.7\% (95\% CI, 86.6\%-96.8\%), and $91.9 \%$ (95\% CI, 88.6\%-95.2\%), respectively. For T-non pCR, B-non pCR, and N-non pCR, the 5-year OS rates were $73.6 \%$ (95\% CI, 68.7\%-78.5\%), $78.9 \%$ $(95 \% \mathrm{CI}, \quad 75.2 \%-82.6 \%)$, and $74.7 \% \quad(95 \% \mathrm{CI}$, 70.0\%-79.4\%), respectively ( $\mathrm{P}<0.0001)$.

According to various intrinsic subtypes, a significantly better 5-year DFS was found in B-pCR patients than in B-non-pCR patients in all intrinsic subtypes except HR(+)HER2(-) ( $\mathrm{P}=0.177$, Figure 3$)$. The differences in 5-year OS between B-pCR and $B-n o n$ pCR in various subtypes are shown in Supplementary Figure 1A. N-pCR patients had significantly better 5-year DFS and 5-year OS than $\mathrm{N}$-non-pCR patients in all subtypes, including the HR(+)HER2 $(-)$ subtype $(\mathrm{P}=0.014$ and 0.027, respectively; Figure 4 and Supplementary Figure 1B).

The univariate and multivariate Cox proportional hazards models of DFS, adjusted for response are summarized in Table 3 and for OS in Table 4. With respect to DFS, the following factors were associated with an increased risk of recurrence: higher T stage, cN2 disease, high histologic grade, no hormonal therapy, and non-pCR. For OS, the risk factors of increased death were higher $\mathrm{T}$ stage, cN2 disease, high histologic grade, no hormonal therapy, and non-pCR. Compared with T-non pCR, T-pCR patients had a lesser risk of recurrence (hazard ratio 0.35, 95\% CI: 0.22-0.56, $\mathrm{P}<0.0001$ ) and death (hazard ratio $0.37,95 \% \mathrm{CI}: 0.19-0.72, \mathrm{P}=0.001)$. The same trends were found either in B-pCR or N-pCR patients. Patients with B-non $\mathrm{pCR}$ had a lower risk of recurrence (hazard ratio 0.81, 95\% CI: 0.66-0.99, $\mathrm{P}=0.041)$ than those with non-pCR, although they lost a significant difference on multivariate analysis; further, there was a non-significant trend of favorable OS in B-non pCR than in T-non pCR. The N-non pCR and T-non pCR patients had the worst 5-year DFS and OS rates. 

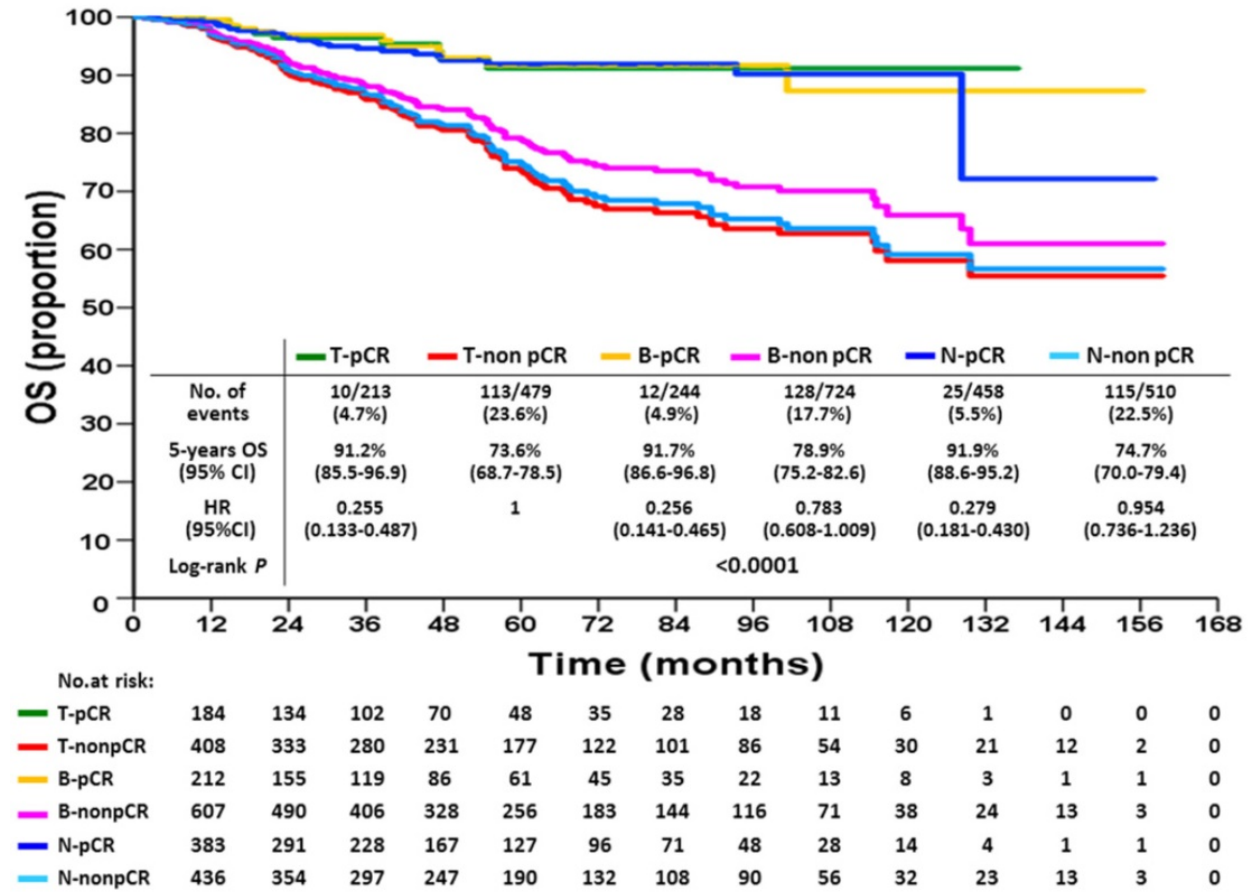

$\begin{array}{cccccccccccccc}184 & 134 & 102 & 70 & 48 & 35 & 28 & 18 & 11 & 6 & 1 & 0 & 0 & 0 \\ 408 & 333 & 280 & 231 & 177 & 122 & 101 & 86 & 54 & 30 & 21 & 12 & 2 & 0 \\ 212 & 155 & 119 & 86 & 61 & 45 & 35 & 22 & 13 & 8 & 3 & 1 & 1 & 0 \\ 607 & 490 & 406 & 328 & 256 & 183 & 144 & 116 & 71 & 38 & 24 & 13 & 3 & 0 \\ 383 & 291 & 228 & 167 & 127 & 96 & 71 & 48 & 28 & 14 & 4 & 1 & 1 & 0 \\ 436 & 354 & 297 & 247 & 190 & 132 & 108 & 90 & 56 & 32 & 23 & 13 & 3 & 0\end{array}$

Figure 2. Overall survival according to breast or axillary node response status after neoadjuvant chemotherapy

HR(+)HER2(-)

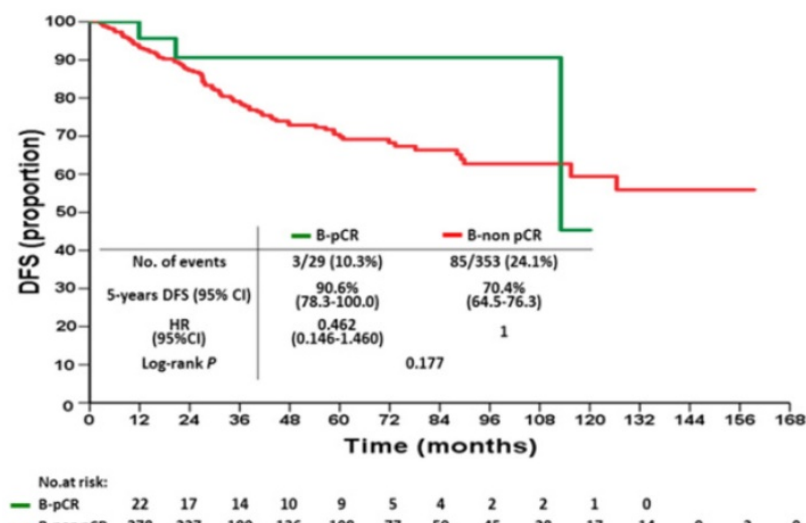

HR(-)HER2(+)

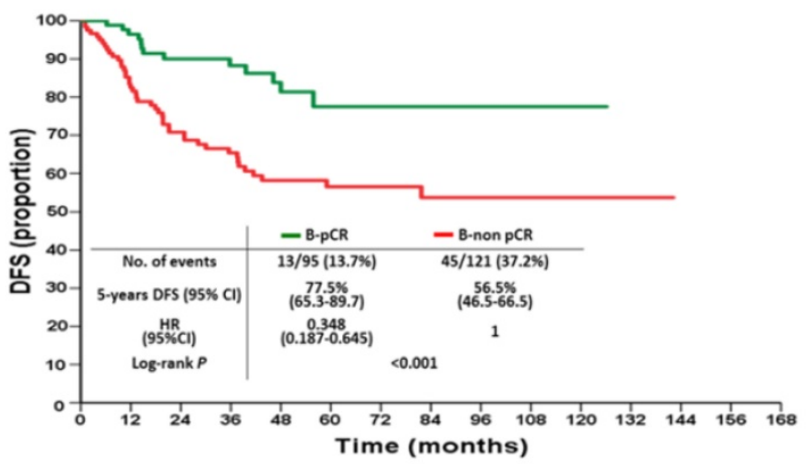

$\begin{array}{ccccccccccccc}\text { No.at risk: } & & & & & & & & & & & \\ \text { - B-pCR } & 80 & 61 & 48 & 33 & 20 & 16 & 12 & 8 & 5 & 4 & 0 & \\ \text { - B-non PCR } & 91 & 68 & 56 & 43 & 33 & 25 & 18 & 16 & 7 & 3 & 2 & 0\end{array}$
HR(+)HER2(+)
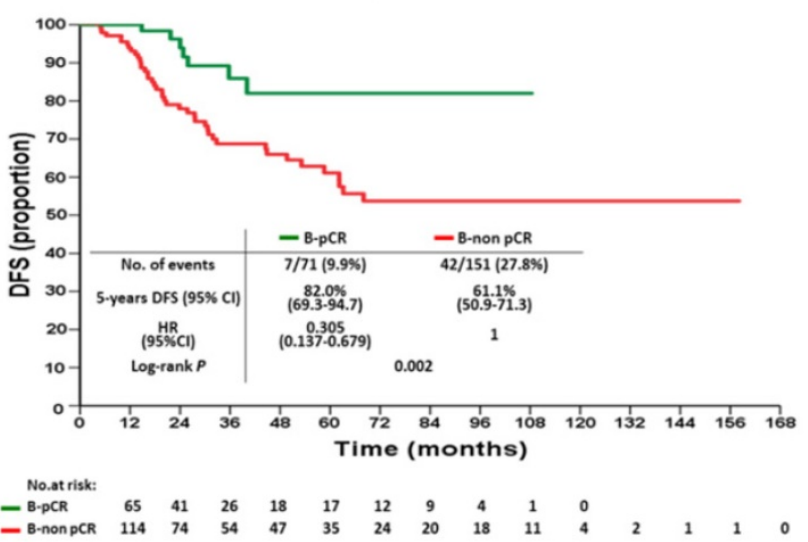

HR(-)HER2(-)
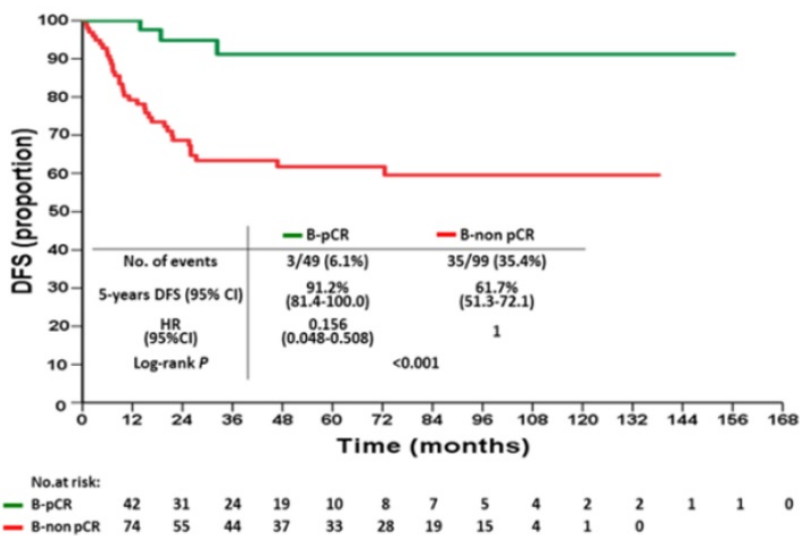

Figure 3. Disease-free survival of $B-p C R$ and $B-n o n ~ p C R$ patients after neoadjuvant chemotherapy by intrinsic subtypes 
HR(+)HER2(-)

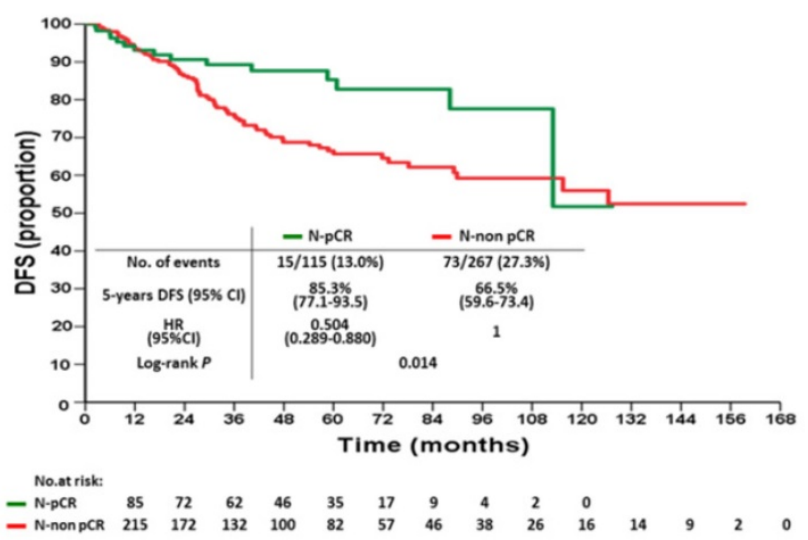

HR(-)HER2(+)

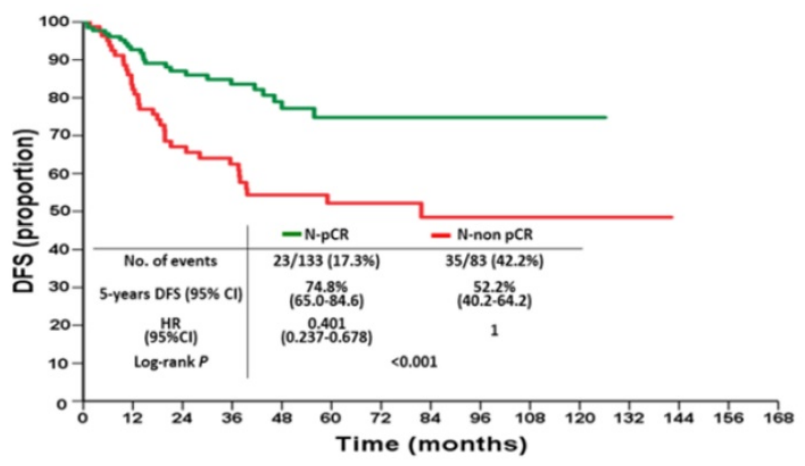

$\begin{array}{ccccccccccccc}\text { No.at risk: } & & & & & & & & & & & \\ - \text { N-pCR } & 107 & 84 & 65 & 44 & 29 & 22 & 18 & 9 & 4 & 0 & & \\ - \text { N-non pCR } & 64 & 45 & 39 & 32 & 24 & 19 & 12 & 10 & 3 & 3 & 2 & 0\end{array}$
HR(+)HER2(+)

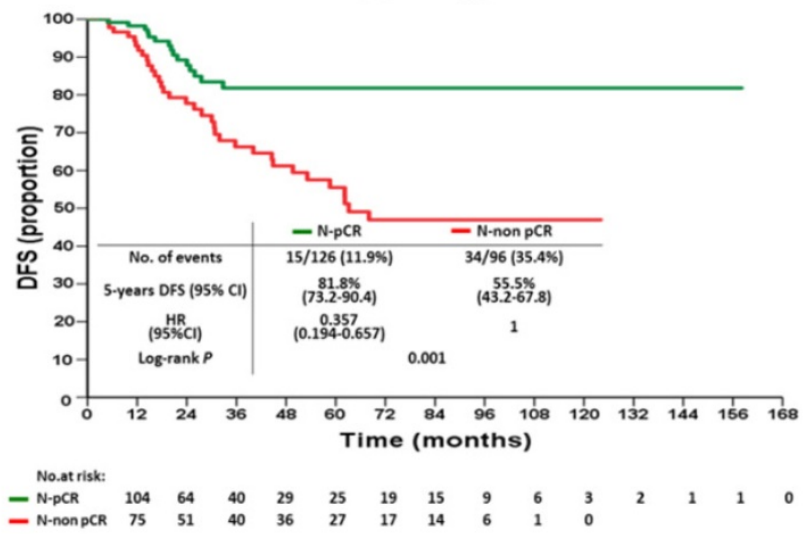

HR(-)HER2(-)

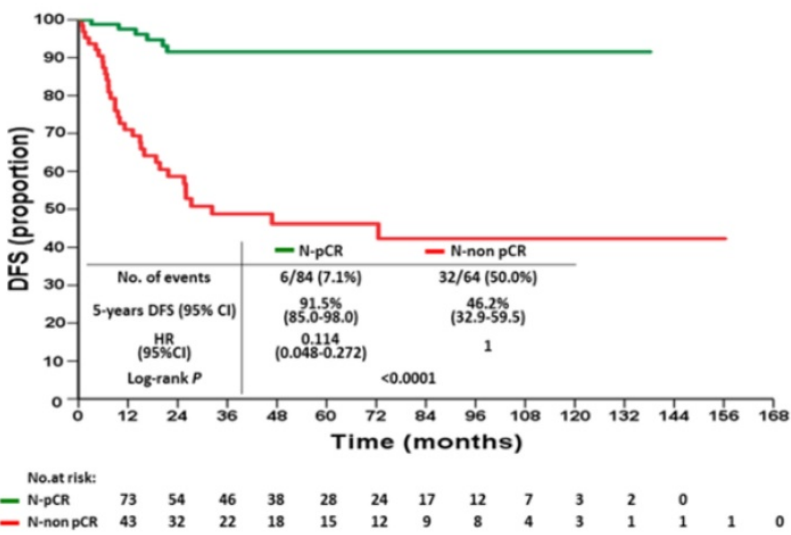

Figure 4. Disease-free survival of $N-p C R$ and $N$-non $p C R$ patients after neoadjuvant chemotherapy by intrinsic subtypes

Table 3. Univariate and multivariate analysis of factors influencing the disease-free survival

\begin{tabular}{|c|c|c|c|c|}
\hline \multirow[t]{2}{*}{ Parameter } & \multicolumn{2}{|l|}{ Univariate } & \multicolumn{2}{|l|}{ Multivariate } \\
\hline & $\begin{array}{l}\text { Hazard ratio }(95 \% \\
\mathrm{CI})\end{array}$ & $P$ value & $\begin{array}{l}\text { Hazard ratio }(95 \% \\
\mathrm{CI})\end{array}$ & $P$ value \\
\hline \multicolumn{5}{|l|}{ Age (yrs) } \\
\hline$\leqq 40$ & Reference & & & \\
\hline $41-60$ & $0.892(0.737-1.080)$ & 0.241 & - & \\
\hline$>60$ & $0.907(0.698-1.180)$ & 0.468 & & \\
\hline \multicolumn{5}{|l|}{ Clinical T stage } \\
\hline $\mathrm{T} 4$ & Reference & & Reference & \\
\hline $\mathrm{T} 1$ & $0.377(0.236-0.601)$ & $<0.001$ & $0.602(0.373-0.974)$ & 0.039 \\
\hline $\mathrm{T} 2$ & $0.444(0.373-0.529)$ & $<0.001$ & $0.603(0.498-0.731)$ & $<0.001$ \\
\hline T3 & $0.555(0.450-0.686)$ & $<0.001$ & $0.724(0.581-0.901)$ & 0.004 \\
\hline \multicolumn{5}{|l|}{ Clinical N stage } \\
\hline $\mathrm{N} 2$ & Reference & & Reference & \\
\hline N1 & $0.336(0.284-0.397)$ & $<0.001$ & $0.473(0.394-0.569)$ & $<0.001$ \\
\hline \multicolumn{5}{|l|}{ Operation type } \\
\hline Mastectomy & Reference & & Reference & \\
\hline BCS & $0.632(0.530-0.755)$ & $<0.001$ & $1.102(0.903-1.345)$ & 0.340 \\
\hline \multicolumn{5}{|l|}{ Histologic grade } \\
\hline 3 & Reference & & Reference & \\
\hline 1 & $0.455(0.311-0.665)$ & $<0.001$ & $0.468(0.317-0.693)$ & $<0.001$ \\
\hline 2 & $0.902(0.766-1.062)$ & 0.215 & $0.936(0.789-1.110)$ & 0.447 \\
\hline \multicolumn{5}{|l|}{ Subtypes } \\
\hline HR(-) HER2(+) & Reference & & Reference & \\
\hline HR(+) HER2(-) & $0.813(0.667-0.990)$ & 0.040 & $1.501(1.091-2.066)$ & 0.013 \\
\hline HR $(+)$ HER2 $(+)$ & $0.890(0.708-1.118)$ & 0.317 & $1.840(1.356-2.496)$ & $<0.001$ \\
\hline HR(-) HER2(-) & $0.992(0.777-1.266)$ & 0.949 & $0.962(0.750-1.233)$ & 0.758 \\
\hline $\begin{array}{l}\text { Pathological } \\
\text { response }\end{array}$ & & & & \\
\hline
\end{tabular}

\begin{tabular}{|c|c|c|c|c|}
\hline \multirow[t]{2}{*}{ Parameter } & \multicolumn{2}{|l|}{ Univariate } & \multicolumn{2}{|l|}{ Multivariate } \\
\hline & $\begin{array}{l}\text { Hazard ratio }(95 \% \\
\mathrm{CI})\end{array}$ & $P$ value & $\begin{array}{l}\text { Hazard ratio (95\% } \\
\text { CI) }\end{array}$ & $P$ value \\
\hline T-non pCR & Reference & & Reference & \\
\hline T-pCR & $0.279(0.177-0.439)$ & $<0.001$ & $0.350(0.218-0.564)$ & $<0.001$ \\
\hline B-pCR & $0.297(0.196-0.448)$ & $<0.001$ & $0.368(0.238-0.568)$ & $<0.001$ \\
\hline B-non pCR & $0.809(0.661-0.992)$ & 0.041 & $0.899(0.732-1.103)$ & 0.307 \\
\hline N-pCR & $0.364(0.271-0.490)$ & $<0.001$ & $0.486(0.354-0.668)$ & $<0.001$ \\
\hline N-non pCR & $0.960(0.777-1.186)$ & 0.703 & $0.968(0.783-1.196)$ & 0.764 \\
\hline \multicolumn{5}{|c|}{ Hormone therapy } \\
\hline No & Reference & & Reference & \\
\hline yes & $0.684(0.587-0.797)$ & $<0.001$ & $0.414(0.315-0.544)$ & $<0.001$ \\
\hline \multicolumn{5}{|c|}{ Radiotherapy } \\
\hline Yes & Reference & & - & \\
\hline No & $0.925(0.772-1.108)$ & 0.399 & & \\
\hline \multicolumn{5}{|c|}{$\begin{array}{l}\text { Abbreviations: CI: confidence interval; BCS: breast-conserving surgery; HR: } \\
\text { hormone receptor; HER2: human epidermal growth factor receptor 2; T-non pCR: } \\
\text { breast and axillary node pathologic non-complete response; T-pCR: breast and } \\
\text { axillary node pathological complete response; B-pCR: breast pathologic complete } \\
\text { response; B-non pCR: breast pathologic non-complete response; N-pCR: axillary } \\
\text { node pathologic complete response; N-non pCR: axillary node pathologic } \\
\text { non-complete response. }\end{array}$} \\
\hline
\end{tabular}

\section{Discussion}

In this retrospective analysis from a single hospital, we demonstrated significantly higher $\mathrm{N}$-pCR rates than B-pCR in all subtypes after NAC, and only a small proportion of N-non-pCR cases $(10.3 \%-16.3 \%)$ were found in these patients. In contrast, our study showed very high $\mathrm{N}$-pCR rates 
(39.7\%-43.4\%) among B-non-pCR patients in all subtypes except HR(+)HER2(-) (25.2\%). The study also confirmed that pCR in the breast, axillary node, or both was associated with improved DFS and OS compared with those with residual cancer. In patients with residual disease, T-non $\mathrm{pCR}$ and $\mathrm{N}$-non pCR had worse DFS than B-non-pCR.

Table 4. Univariate and multivariate analysis of factors influencing the overall survival

\begin{tabular}{|c|c|c|c|c|}
\hline \multirow[t]{2}{*}{ Parameter } & \multicolumn{2}{|l|}{ Univariate } & \multicolumn{2}{|l|}{ Multivariate } \\
\hline & $\begin{array}{l}\text { Hazard ratio }(95 \% \\
\mathrm{CI})\end{array}$ & $P$ value & $\begin{array}{l}\text { Hazard ratio }(95 \% \\
\mathrm{CI})\end{array}$ & $P$ value \\
\hline \multicolumn{5}{|l|}{ Age (yrs) } \\
\hline$\leqq 40$ & Reference & & & \\
\hline $41-60$ & $0.897(0.703-1.144)$ & 0.380 & - & \\
\hline$>60$ & 0.987 (0.707-1.379) & 0.939 & & \\
\hline \multicolumn{5}{|l|}{ Clinical T stage } \\
\hline $\mathrm{T} 4$ & Reference & & Reference & \\
\hline $\mathrm{T} 1$ & $0.242(0.119-0.492)$ & $<0.001$ & $0.460(0.223-0.948)$ & 0.035 \\
\hline $\mathrm{T} 2$ & $0.335(0.267-0.421)$ & $<0.001$ & $0.477(0.372-0.611)$ & $<0.001$ \\
\hline T3 & $0.512(0.398-0.660)$ & $<0.001$ & $0.669(0.513-0.871)$ & 0.003 \\
\hline \multicolumn{5}{|l|}{ Clinical N stage } \\
\hline $\mathrm{N} 2$ & Reference & & Reference & \\
\hline N1 & $0.315(0.252-0.395)$ & $<0.001$ & $0.533(0.416-0.682)$ & $<0.001$ \\
\hline \multicolumn{5}{|l|}{ Operation type } \\
\hline Mastectomy & Reference & & Reference & \\
\hline BCS & $0.419(0.320-0.549)$ & $<0.001$ & $0.776(0.577-1.044)$ & 0.094 \\
\hline \multicolumn{5}{|l|}{ Histologic grade } \\
\hline 3 & Reference & & Reference & \\
\hline 1 & $0.365(0.216-0.616)$ & $<0.001$ & $0.397(0.231-0.682)$ & $<0.001$ \\
\hline 2 & $0.933(0.759-1.147)$ & 0.511 & $1.034(0.833-1.283)$ & 0.762 \\
\hline \multicolumn{5}{|l|}{ Subtype } \\
\hline HR(-) HER2(+) & Reference & & Reference & \\
\hline HR(+) HER2(-) & $0.782(0.602-1.015)$ & 0.065 & $1.693(1.158-2.474)$ & 0.007 \\
\hline HR(+) HER2(+) & $0.988(0.738-1.324)$ & 0.937 & $2.194(1.526-3.155)$ & $<0.001$ \\
\hline HR(-) HER2(-) & $1.242(0.919-1.679)$ & 0.158 & $1.248(0.920-1.692)$ & 0.155 \\
\hline \multicolumn{5}{|l|}{$\begin{array}{l}\text { Pathological } \\
\text { response }\end{array}$} \\
\hline T-non pCR & Reference & & Reference & \\
\hline T-PCR & $0.255(0.133-0.487)$ & $<0.001$ & $0.367(0.188-0.718)$ & 0.003 \\
\hline B-pCR & $0.256(0.141-0.465)$ & $<0.001$ & $0.359(0.194-0.667)$ & 0.001 \\
\hline B-non pCR & $0.783(0.608-1.009)$ & 0.059 & $0.873(0.677-1.127)$ & 0.297 \\
\hline N-pCR & $0.279(0.181-0.430)$ & $<0.001$ & $0.411(0.261-0.648)$ & $<0.001$ \\
\hline N-non pCR & $0.954(0.736-1.236)$ & 0.720 & $0.965(0.744-1.252)$ & 0.789 \\
\hline \multicolumn{5}{|l|}{ Hormone therapy } \\
\hline No & Reference & & Reference & \\
\hline Yes & $0.575(0.472-0.700)$ & $<0.001$ & $0.357(0.259-0.492)$ & $<0.001$ \\
\hline \multicolumn{5}{|l|}{ Radiotherapy } \\
\hline Yes & Reference & & - & \\
\hline No & $1.065(0.852-1.333)$ & 0.579 & & \\
\hline \multicolumn{5}{|c|}{$\begin{array}{l}\text { Abbreviations: CI: confidence interval; BCS: breast-conserving surgery; HR: } \\
\text { hormone receptor; HER2: human epidermal growth factor receptor 2; T-non pCR: } \\
\text { breast and axillary node pathologic non-complete response; T-pCR: breast and } \\
\text { axillary node pathological complete response; B-pCR: breast pathologic complete } \\
\text { response; B-non pCR: breast pathologic non-complete response; N-pCR: axillary } \\
\text { node pathologic complete response; N-non pCR: axillary node pathologic } \\
\text { non-complete response. }\end{array}$} \\
\hline
\end{tabular}

Positive axillary lymph node status is a more important prognostic factor than tumor size in early breast cancer and remains the worst prognostic indicator following NAC. There is much evidence suggesting that metastatic lymph nodes need to be treated because cancer cells in lymph nodes can exit and spread to distant metastatic sites. The lymph node window model experiment showed that cancer cells in lymph nodes cause lymphatic vessel regression and lack of angiogenesis in the formation of lymph node metastasis [17], which induces poor control and difficulty in eradicating the disease in metastatic lymph nodes. Inconsistent results and very high alterations in both estrogen receptor and HER2 status were observed throughout the process of tumor progression.[18] Furthermore, different clone mutations in the axillary node with additional copy number changes were demonstrated in a genomic study using single-cell sequencing [19], and few new mutations were not observed in the primary tumor with key driver mutation in synchronous lymph node metastasis.[20] These findings indicate difficulty in treating metastatic axillary nodes compared with breast tumors. However, many studies, including ours, have shown very high N-pCR rates (23\%-61\%).[21] The paradoxical findings of difficult treatment and good axillary nodal response rate to NAC cannot simply be explained by the biological nature of the disease or genomic findings. The relatively smaller metastatic tumor volume within the axillary node in comparison to the breast tumor itself should be one of the predictive factors. The difference in $\mathrm{N}$-pCR rates between $\mathrm{cN} 1$ and $\mathrm{cN} 2$ disease in our study confirmed that the difference in tumor size burden of metastatic nodes is one of the contributing factors of N-pCR (Supplementary Table 1A, 1B). Through literatures reviewed, relative low N-pCR rates (around 20\%) were reported with conventional chemotherapy 2 decades ago [22, 23], while much higher N-pCR rates after incorporating intensive chemotherapy and targeted therapy were reported in the last decade. [24, 25] Our data and others [26] showed that there were very high N-pCR rates in this cohort. Similarly, high N-pCR rates (25.2\%-43.4\%) were found even in B-non pCR patients in various intrinsic subtypes. The high N-pCR rates found in B-non-pCR patients have also been poorly explored. Besides the smaller tumor burden in axillary nodes, the observation that the cancericidal effect of concurrent novel agents such as trastuzumab and intensive chemotherapy occurred mainly on metastatic lymph nodes rather than the tumor itself was a possible explanation.

NAC for operable breast cancer can eliminate axillary nodal metastasis to avoid axillary dissection, and several prediction models on this have been developed.[12, 27-29] One prospective and another retrospective study found that $\mathrm{N}-\mathrm{pCR}$ is highly correlated with B-pCR, and nodal positivity rates were $<2 \%$ after NAC in HER2 $(+)$ and triple-negative breast cancer. This provided the rationale for omitting axillary surgery in specific subtypes while B-pCR was achieved.[11, 30] Although high incidence rates 
(83.7\%-89.7\%) of $\mathrm{N}-\mathrm{pCR}$ were found in B-pCR patients in our study, they did not support the hypothesis of de-escalating axillary surgery if B-pCR was confirmed after minimally invasive biopsy, because worse DFS was found in patients with residual axillary node disease after NAC compared with residual disease in the breast. This finding underlines the prognostic relevance of pathologic nodal status after NAC, which is consistent with recent data suggesting that sentinel node biopsy should not be routinely substituted for axillary dissection with ypN1 disease.[31]

Even if the improvement of event-free survival (EFS) could not be established with pCR in clinical trial level analysis [1], many updated studies reported the correlation between pCR and DFS and OS in most subtypes regardless of the chemotherapy regimens administered. [5, 32, 33] A recent meta-analysis including 52 trials also demonstrated that patients with pCR after NAC had significantly better EFS (hazard ratio 0.31, 95\% CI: 0.24-0.39) and had associated improved survival (hazard ratio 0.22, 95\% CI: 0.15-0.31) except for the HR(+)HER2(-) subtype.[2] Our study confirmed that patients with pCR, regardless of the breast, axillary node, or both, had better DFS and OS than non-pCR patients in all intrinsic subtypes except HR(+)HER2(-).

Worse outcomes were found in those with residual disease after NAC in most studies, although whether B-non pCR or non-pCR is a more important prognostic factor for poor survival has been rarely studied. Two reports showed that the worst outcomes occurred with N-non-pCR in the HR(-)HER2(-) subtype.[7, 19] In our study, patients with residual disease in either the breast or axillary node had worse outcomes. The worst 5-year DFS was found in non-pCR patients with $\mathrm{HR}(+) \mathrm{HER} 2(+)$, HR(-)HER2(+), and HR(-)HER2(-) subtypes (55.5\%, $52.2 \%$, and $46.2 \%$, respectively), and the worst 5 -year OS rates were found in the N-non pCR of HR(-)HER2(+) and HR(-)HER2(-)subtypes (64.8\% and $56.4 \%$, respectively) (Supplementary Figure 1B). This finding suggests that searching for more effective drugs to eradicate cancer cells in metastatic axillary nodes rather than the breast tumor itself is crucial when designing NAC clinical trials. Therefore, the accurate selection of optimal patients with tailor-appropriate neoadjuvant regimens will improve the N-pCR rate and de-escalate axillary surgery.

As mentioned above, the response of breast tumors or axillary nodes to chemotherapy or novel agents vary. The residual cancer burden (RCB) is one of the standard classifications of the response to NAC, which is based on four parameters of combined response, including breast tumor and axillary node response to conventional chemotherapy regimens.[17] Our data are consistent with the retrospective studies from a single institute [5] and National Cancer Database [7], which found that the response to chemotherapy and correlation of survival to pCR can be based on the breast and axillary nodes separately, or the combined treatment responses of breast and axillary nodes together, such as in RCB.

\section{Limitations}

The retrospective analysis is one of the limitations of this study, as well as its shorter follow-up time. Meanwhile, not all HER2 positive cancers received trastuzumab-containing anti-HER2 therapy in our cohort. Although the use of trastuzumab for HER2-positive breast cancer had become standard of care since 2005, it was reimbursed as adjuvant or neoadjuvant therapy since 2010 .

\section{Conclusions}

This study demonstrates that N-pCR is highly correlated with B-pCR in all intrinsic subtypes of clinically node-positive breast cancer after NAC, and that the biologic heterogeneity of discrepancies between B-pCR and N-pCR rates to different treatment regimens needs further exploration. Our data did not support the omission of axillary surgery because worse DFS was found in patients with residual axillary node disease after NAC than those with residual breast disease.

\section{Abbreviations}

B-pCR: breast pathologic complete response; N-pCR: axillary node pathologic complete response; NAC: neoadjuvant chemotherapy; T-pCR: total pathologic complete response; N-non pCR: axillary node pathologic non-complete response; B-non $\mathrm{pCR}$ : breast pathologic non-complete response; T-non pCR: total pathologic non-complete response; HR: hormone receptor; HER2: human epidermal growth factor receptor 2; OS: overall survival; pCR: pathologic complete response; DFS: disease-free survival; $\mathrm{cN}+$ : clinically node-positive; cN0: clinically node-negative; IHC: immunohistochemistry; FISH: fluorescence in situ hybridization; IQRs: interquartile ranges; KM: Kaplan-Meier; CIs: confidence intervals; EFS: event-free survival.

\section{Supplementary Material}

Supplementary figures and tables. http://www.jcancer.org/v12p5365s1.pdf 


\section{Acknowledgments}

\section{Funding}

This research did not receive any specific grant from funding agencies in the public, commercial, or not-for-profit sectors.

\section{Ethics approval}

The study was approved by the Chang Gung Foundation Institutional Review Board (IRB No. 201601335B0). The ethics committees waived the need for written informed consent because no intervention was involved and the retrospective analysis of clinical data.

\section{Competing Interests}

The authors have declared that no competing interest exists.

\section{References}

1. Cortazar P, Zhang L, Untch M, Mehta K, Costantino JP, Wolmark N, et al. Pathological complete response and long-term clinical benefit in breast cancer: the CTNeoBC pooled analysis. Lancet. 2014;384:164-72

2. Spring LM, Fell G, Arfe A, Sharma C, Greenup RA, Reynolds KL, et al. Pathological complete response after neoadjuvant chemotherapy and impact on breast cancer recurrence and survival: a comprehensive meta-analysis. Clin Cancer Res. 2020;26:2838-48

3. Berruti A, Amoroso V, Gallo F, Bertaglia V, Simoncini E, Pedersini R, et al. Pathologic complete response as a potential surrogate for the clinical outcome in patients with breast cancer after neoadjuvant therapy: a meta-regression of 29 randomized prospective studies. J Clin Oncol. 2014;32:3883-91

4. Wang-Lopez Q, Chalabi N, Abrial C, Radosevic-Robin N, Durando X, Mouret-Reynier MA, et al. Can pathologic complete response (pCR) be used as a surrogate marker of survival after neoadjuvant therapy for breast cancer? Crit Rev Oncol Hematol. 2015;95:88-104

5. Mougalian SS, Hernandez M, Lei X, Lynch S, Kuerer HM, Symmans WF, et al. Ten-Year Outcomes of Patients With Breast Cancer With Cytologically Confirmed Axillary Lymph Node Metastases and Pathologic Complete Response After Primary Systemic Chemotherapy. JAMA Oncol. 2016;2:508-16

6. Symmans WF, Wei C, Gould R, Yu X, Zhang Y, Liu M, et al. Long-Term Prognostic Risk After Neoadjuvant Chemotherapy Associated With Residual Cancer Burden and Breast Cancer Subtype. J Clin Oncol. 2017;35:1049-60

7. Boughey JC, Ballman KV, McCall LM, Mittendorf EA, Symmans WF, Julian TB, et al. Tumor Biology and Response to Chemotherapy Impact Breast Cancer-specific Survival in Node-positive Breast Cancer Patients Treated With Neoadjuvant Chemotherapy: Long-term Follow-up From ACOSOG Z1071 (Alliance). Ann Surg. 2017;266:667-76

8. Kuerer HM, Rauch GM, Krishnamurthy $S$, Adrada BE, Caudle AS, DeSnyder SM, et al. A Clinical Feasibility Trial for Identification of Exceptional Responders in Whom Breast Cancer Surgery Can Be Eliminated Following Neoadjuvant Systemic Therapy. Ann Surg. 2018;267:946-51

9. Simons JM, Koppert LB, Luiten EJT, van der Pol CC, Samiei S, de Wilt JHW, et al. De-escalation of axillary surgery in breast cancer patients treated in the neoadjuvant setting: a Dutch population-based study. Breast Cancer Res Treat. 2020;180:725-33

10. Bertucci F, Ng CKY, Patsouris A, Droin N, Piscuoglio S, Carbuccia N, et al. Genomic characterization of metastatic breast cancers. Nature. 2019;569:560-4

11. Tadros AB, Yang WT, Krishnamurthy S, Rauch GM, Smith BD, Valero V, et al. Identification of Patients With Documented Pathologic Complete Response in the Breast After Neoadjuvant Chemotherapy for Omission of Axillary Surgery. JAMA Surg. 2017;152:665-70

12. Kantor O, Sipsy LM, Yao K, James TA. A Predictive Model for Axillary Node Pathologic Complete Response after Neoadjuvant Chemotherapy for Breast Cancer. Ann Surg Oncol. 2018;25:1304-11
13. Kozak MM, Jacobson CE, von Eyben R, Pollom EL, Telli M, Horst KC. Outcomes Following Neoadjuvant Chemotherapy for Breast Cancer in Women Aged 40 Years and Younger: Impact of Pathologic Nodal Response. J Natl Compr Canc Netw. 2018;16:845-50

14. Fleming CA, McCarthy K, Ryan C, McCarthy A, O'Reilly S, O'Mahony D, et al. Evaluation of Discordance in Primary Tumor and Lymph Node Response After Neoadjuvant Therapy in Breast Cancer. Clin Breast Cancer. 2018;18:e255-e61

15. Broglio KR, Quintana M, Foster M, Olinger M, McGlothlin A, Berry SM, et al. Association of Pathologic Complete Response to Neoadjuvant Therapy in HER2-Positive Breast Cancer With Long-Term Outcomes: A Meta-Analysis. JAMA Oncol. 2016;2:751-60

16. Symmans WF, Peintinger F, Hatzis C, Rajan R, Kuerer $H$, Valero V, et al. Measurement of residual breast cancer burden to predict survival after neoadjuvant chemotherapy. J Clin Oncol. 2007;25:4414-22

17. Jeong HS, Jones D, Liao S, Wattson DA, Cui CH, Duda DG, et al. Investigation of the Lack of Angiogenesis in the Formation of Lymph Node Metastases. J Natl Cancer Inst. 2015;107:djv155

18. Lindström LS, Karlsson E, Wilking UM, Johansson U, Hartman J, Lidbrink EK, et al. Clinically used breast cancer markers such as estrogen receptor, progesterone receptor, and human epidermal growth factor receptor 2 are unstable throughout tumor progression. J Clin Oncol. 2012;30:2601-8

19. Bao L, Qian Z, Lyng MB, Wang L, Yu Y, Wang T, et al. Coexisting genomic aberrations associated with lymph node metastasis in breast cancer. J Clin Invest. 2018;128:2310-24

20. Yates LR, Knappskog S, Wedge D, Farmery JHR, Gonzalez S, Martincorena I, et al. Genomic Evolution of Breast Cancer Metastasis and Relapse. Cancer Cell. 2017;32:169-84.e7

21. Mamtani A, Barrio AV, King TA, Van Zee KJ, Plitas G, Pilewskie M, et al. How Often Does Neoadjuvant Chemotherapy Avoid Axillary Dissection in Patients With Histologically Confirmed Nodal Metastases? Results of a Prospective Study. Ann Surg Oncol. 2016;23:3467-74

22. Rouzier R, Extra JM, Klijanienko J, Falcou MC, Asselain B, Vincent-Salomon A, et al. Incidence and prognostic significance of complete axillary downstaging after primary chemotherapy in breast cancer patients with T1 to T3 tumors and cytologically proven axillary metastatic lymph nodes. J Clin Oncol. 2002;20:1304-10

23. Hennessy BT, Hortobagyi GN, Rouzier R, Kuerer H, Sneige N, Buzdar AU, et al. Outcome after pathologic complete eradication of cytologically proven breast cancer axillary node metastases following primary chemotherapy. J Clin Oncol. 2005;23:9304-11

24. Gianni L, Pienkowski T, Im YH, Roman L, Tseng LM, Liu MC, et al. Efficacy and safety of neoadjuvant pertuzumab and trastuzumab in women with locally advanced, inflammatory, or early HER2-positive breast cancer (NeoSphere): a randomised multicentre, open-label, phase 2 trial. Lancet Oncol. 2012;13:25-32

25. Schneeweiss A, Chia S, Hickish T, Harvey V, Eniu A, Hegg R, et al. Pertuzumab plus trastuzumab in combination with standard neoadjuvant anthracycline-containing and anthracycline-free chemotherapy regimens in patients with HER2-positive early breast cancer: a randomized phase II cardiac safety study (TRYPHAENA). Ann Oncol. 2013;24:2278-84

26. Samiei S, van Nijnatten TJA, de Munck L, Keymeulen K, Simons JM, Kooreman LFS, et al. Correlation Between Pathologic Complete Response in the Breast and Absence of Axillary Lymph Node Metastases After Neoadjuvant Systemic Therapy. Ann Surg. 2020;271:574-80

27. Vila J, Mittendorf EA, Farante G, Bassett RL, Veronesi P, Galimberti V, et al. Nomograms for Predicting Axillary Response to Neoadjuvant Chemotherapy in Clinically Node-Positive Patients with Breast Cancer. Ann Surg Oncol. 2016;23:3501-9

28. Ouldamer L, Chas M, Arbion F, Body G, Cirier J, Ballester M, et al. Risk scoring system for predicting axillary response after neoadjuvant chemotherapy in initially node-positive women with breast cancer. Surg Oncol. 2018;27:158-65

29. Simons JM, van Nijnatten TJA, van der Pol CC, Luiten EJT, Koppert LB, Smidt ML. Diagnostic Accuracy of Different Surgical Procedures for Axillary Staging After Neoadjuvant Systemic Therapy in Node-positive Breast Cancer: A Systematic Review and Meta-analysis. Ann Surg. 2019;269:432-42

30. Barron AU, Hoskin TL, Day CN, Hwang ES, Kuerer HM, Boughey JC. Association of Low Nodal Positivity Rate Among Patients With ERBB2-Positive or Triple-Negative Breast Cancer and Breast Pathologic Complete Response to Neoadjuvant Chemotherapy. JAMA Surg. 2018;153:1120-6

31. Almahariq MF, Levitin R, Quinn TJ, Chen PY, Dekhne N, Kiran S, et al. Omission of Axillary Lymph Node Dissection is Associated with Inferior Survival in Breast Cancer Patients with Residual N1 Nodal Disease Following Neoadjuvant Chemotherapy. Ann Surg Oncol. 2021;28:930-40 
32. Fayanju OM, Ren Y, Thomas SM, Greenup RA, Plichta JK, Rosenberger $\mathrm{LH}$, et al. The Clinical Significance of Breast-only and Node-only Pathologic Complete Response (pCR) After Neoadjuvant Chemotherapy (NACT): A Review of 20,000 Breast Cancer Patients in the National Cancer Data Base (NCDB). Ann Surg. 2018;268:591-601

33. Weiss A, Bashour SI, Hess K, Thompson AM, Ibrahim NK. Effect of neoadjuvant chemotherapy regimen on relapse-free survival among patients with breast cancer achieving a pathologic complete response: an early step in the de-escalation of neoadjuvant chemotherapy. Breast Cancer Res. 2018;20:27 